\title{
In Situ Synthesis of Polyaniline Based Ceramic Wastes Composites: Dielectric and Electrical Properties
}

\section{Sohail ( $\sim$ msohail2000@gmail.com )}

University of Swat

Adnan Shahzad

University of Swat

Mian Gul Sayed

University of Swat

Ihsan Ullah

University of Swat

M. Omer

University of Swat

Adnan Adnan

University of Swat

Fazal Mabood

University of Swat

Aziz Ahmad

University of Swat

\section{Research Article}

Keywords: Waste ceramic material, polymer composite, SEM, dielectric properties

Posted Date: November 30th, 2021

DOI: https://doi.org/10.21203/rs.3.rs-1111541/v1

License: (1) (1) This work is licensed under a Creative Commons Attribution 4.0 International License. Read Full License 


\section{Abstract}

In the present study, ceramic wastes collected from the premises of industrial zone in Peshawar, KP Pakistan were investigated. An effort has been made to recycle and use the ceramic wastes as fillers in polymeric composites. The negative cost ceramic wastes were purified and activated thermally. The elemental composition and pellets of the wastes were investigated through SEM/EDX analysis. Waste/Polyaniline (PANI) composite was synthesized via in-situ free radical polymerization technique. SEM of the composites showed the uniform distribution of fillers particles in the PANI matrix. XRD studies confirmed that the prepared composite material had a face- centered cubic geometry with distinct preferential orientations. Dielectric analysis showed that the materials exhibit active performance at high frequency regions $(3 \mathrm{MHz}$ to $3 \mathrm{GHz})$ at room temperature. The results show decrease in dielectric losses and capacitance $(1.6 \mathrm{pF})$ at high frequency regions. AC conductivity of the composite has been increased up to $37.95 \mathrm{Scm}^{-1}$. This revealed the effect of PANI on the ceramic wastes while increasing its conductance performance. This suggests that the composite material can be investigated for use in photovoltaic detectors, electro-responsive capacitors and power applications.

\section{Introduction}

Wastes generated due to human and industrial activities produce massive pollution thus affecting our environment badly. Industries make available goods, jobs and services while contributing in environmental pollution and wastes on the other side. Industrial pollution has many features. It infects many sources of drinking water (water pollution), discharges surplus toxins into the air (air pollution) and reduces soil quality (soil pollution), wildlife extinction and global warming all over the world. Huge water pollution is also caused by industrial wastes while adding pollutants that are very much harmful for aquatic as well as terrestrial life. Major environmental calamities happened due to industrial mishaps while adding various types of trace metals in river water [1].

The cost of natural resources is increasing with time. Development in material skills is continually decreasing the use of natural capitals thus reducing pollutants load due to which major focus lies now on the recapture, reprocessing of natural capitals and search for more substitutes [2]. Industrial residues are dumped in landfills ignoring their capability for recycling and reprocessing. At present, large amount of ceramic wastes are produced in ceramic industries that have a significant impact on environment and humans. One third (1/3) of the total ceramic production is waste material [3]. Reusing waste materials results in green synthesis, eco-friendly products, green, energy saving, and pollution free environment.

Waste ceramics coming out are usually in the form of powder and tile's pellets. These are generated in the industry during the process of dressing, cutting and polishing. Though a part of these wastes is used near the industrial plants (e.g. in excavated pit refill) however, the remaining part needs open field for disposal. As a result, these wastes disperse in surroundings while indulging the aesthetic scene all around. However, the ceramic waste is durable, hard and highly resistant to biological, chemical, and 
physical changes. With increase in ceramic waste heaping up every day, there is a pressure on ceramic producers for its useful disposal.

China, (world's largest ceramic producers and consumers) yields over one million tons of ceramic wastes annually [4]. These wastes are just dumped or stacked nearby every year. In India, annual ceramic's yield is about 100 million tons of which $15-30 \%$ wastes are generated [5]. A lot of work has been done on the designing of comprehensive ways for the reutilization of ceramic wastes. Ceramic wastes and calcined clays have been recycled as alternatives for cement $[6,7]$. Possible applications of ceramic wastes as a substitute for natural aggregates (gravel or sand) have also been reported [8-11]. Waste ceramics were used for the synthesis of geopolymers [12]. Nano-silica recycled from construction and demolishing waste has been used for the development of construction materials [13] [14]. Waste from electricity distribution networks have been recycled and used as reinforcing materials in polymer composites [15]. Porous ceramic membrane was designed from recycled waste fly ash [16]. Thermal and electrical properties of hybrid materials comprising of conducting PANI based waste mud were investigated [17] [18]. Polymer composite based on waste material was proposed for possible high voltage outdoor application [19]. Physical and mechanical properties of gypsum plaster composites affected by some waste additives have also been studied [20]. PANI based composite materials has been used in electrochemical sensors for the detection of explosives [21]. PANI/zeolite nanocomposites have been investigated as photoelectrode for the catalytic hydrogen production [22]. PANI based composites have also been widely used as adsorbents for the removal of heavy metals from wastewater [23]. However, no work has been done for the use of waste ceramic as filler in PANI matrix for exploring their physical and dielectric properties. Here, we report the first study in this regard.

There are about seven big industries that produce different ceramic based products in Pakistan with an annual installed capacity of 22 million square meter $\left(\mathrm{m}^{2}\right)$ while the production figure is 18.7 million $\mathrm{m}^{2}$ [24]. The waste produced is in the form of powder, pellets and cakes discharged from the plant at various stages which are not recycled in any form. Near the industrial unit, some advised areas are marked for dumping of these wastes. However, most industrial units throw their wastes in ditches or available place carelessly. These misplace dumping cause's immense environmental and land degradation. Therefore, there remains a need for the proper way of disposal of these wastes rapidly. In addition, recycling them for some useful applications is a best strategy. No dedicated work has been done about the systematic characterization and reutilization of these wastes. Herein, waste ceramics found in the local industry site (FORT ceramic industry, Peshawar, Khyber Pakhtunkhwa (KP), Pakistan) have been creatively presented to investigate its portability for polymer composite formation. FORT industry bears one of the largest ceramic manufacturing plant. PANI has been utilized as matrix due its good environmental stability, facile synthesis and electrical conductivity. The whole process was carried out in aqueous media to make it more environmentally friendly. The present study is novel in the sense that this is the first ever work performed in the country Pakistan. Secondly, the technique (in-situ free radical polymerization has been used for the first time in the production of the waste composites and the composites have been studied for the first time for their dielectric and capacitance performance. 


\section{Experimental}

\subsection{Materials}

The ceramic used in the present study was extracted from the wastes collected from inside and surroundings of FORT ceramic industry located in Hayatabad Peshawar Pakistan. For PANI production, aniline (98\%), hydrochloric acid (37\%) and ammonium persulphate (98\%) were purchased from Sharlu (Spain) and were used as received. Millipore deionized water (doubly distilled) was used in the process of polymerization and washing.

\subsection{Devices and equipment}

EDX analysis was performed for the determination of elemental composition of ceramic wastes (CW) by X-ray Philips analytical diffractometer. Morphology and particle size of the CW and CW/PANI composite was investigated by scanning electron microscope (JEOL JSM- 6700F). For XRD analysis, JDX-3532 Xray diffractometer (JEOL JAPAN) with a fixed radiation wavelength of $\lambda-1.54 A^{\circ}$ was used. Dielectric properties of the samples were studied using RF Impedance/Material Analyzer (Agilent E4 997 A) at a temperature of $300 \mathrm{~K}$ with frequency in the range of $1 \mathrm{MHz}-3 \mathrm{GHz}$.

\subsection{Activation of ceramic wastes (CW)}

The obtained ceramic samples were mostly pellets, cakes, precipitate and cracked tiles that were formed during industrial processing. Ultrasonically, waste samples were dipped to wash in refined water in order to remove contaminants for example organic matters, metals and dust etc. For drying, the humid wastes were kept in an electric oven at $60^{\circ} \mathrm{C}$ for about 12 hours. After this time period, dried wastes were then properly powdered in a mortar manually for half an hour and were passed through a mesh of $250 \mu$ pore size.

\subsection{Synthesis of ceramic waste/PANI composite}

Ceramic waste/PANI composite was synthesized via in-situ free radical polymerization route which is a suitable production method for composite materials. Similar technique has been used for the preparation of PANI nanofibers [25], PANI/cellulose nanocrystals composites [26], well defined surface modified PANI nanotubes [27] and PANI/polystyrene composites [28]. A weighed amount (250 mg) of CW was dispersed in $100 \mathrm{ml}$ deionized water under magnetic stirring. A solution of $10 \%$ aniline in $1 \mathrm{M} \mathrm{HCl}$ was prepared from which $25 \mathrm{ml}$ was poured drop wise in $\mathrm{CW}$ parent solution. The whole mixture was magnetically stirred for about 1 hour. Subsequently, ammonium persulphate $\left(\left(\mathrm{NH}_{4}\right)_{2} \mathrm{~S}_{2} \mathrm{O}_{8}\right)(25 \mathrm{ml})$ solution prepared in IM HCl was injected drop wise to parent solution. The whole mixture (system) was kept under magnetic stirring at varying temperature range from $0-5^{\circ} \mathrm{C}$ for about 3 hours with subsequent overnight assimilation. Thereafter, byproducts and impurities in the mixture were removed via centrifugation (4000 rpm) for 10 minutes twice. The precipitated composite was filtered and washed three times with deionized water and was dried in an oven at $70^{\circ} \mathrm{C}$. The obtained composite was abbreviated as CW/PANI. All the polymerization conditions were optimized before obtaining this standard composite sample. 


\section{Results And Discussion}

\subsection{SEM/EDX Analysis}

Elemental composition of the ceramic powder was obtained by using EDX analysis and the results are given in Fig. 1 and Table 1. The main constituents of ceramic powder are silicon, aluminum, calcium, iron and magnesium in the form of their oxides [24].

Table 1

Elemental composition with respect to atomic and weight percent measures of CW.

\begin{tabular}{|lllll|}
\hline S.No & Name of Element & symbol & Atomic \% & Weight \% \\
\hline 1 & Oxygen & $\mathrm{O}$ & 69.72 & 55.14 \\
\hline 2 & Sodium & $\mathrm{Na}$ & 00.31 & 00.35 \\
\hline 3 & Magnesium & $\mathrm{Mg}$ & 02.05 & 02.46 \\
\hline 4 & Aluminum & $\mathrm{Al}$ & 06.48 & 08.64 \\
\hline 5 & Silicon & $\mathrm{Si}$ & 17.04 & 23.65 \\
8 & Potassium & $\mathrm{K}$ & 00.70 & 01.36 \\
9 & Titanium & $\mathrm{Ca}$ & 02.25 & 04.46 \\
\hline
\end{tabular}

Morphology of CW and CW/PANI was analyzed via SEM micrographs as shown in Fig. 2 (a) and (b) respectively. It was found that powder contains entirely irregular, angular pellets and coarse particles. The average particles size measured was $1.75 \mu \mathrm{m}$. The major crystalline phases in the powder sample are seen to be that of $\mathrm{SiO}_{2}$ (quartz) and $\mathrm{NaAlSi}_{3} \mathrm{O}_{8}$ (albite). CW/PANI composite displays smooth morphology which indicates the homogenous diffusion of wastes aggregates in the PANI matrix. This results in the firm immobilization of waste particles over the surface [29]. Such a plane surface of the composite represents its enhanced capacitance characteristics with better cycling stability [30]. It is also apparent that waste particles are not agglomerated and spread in the polymer environment separately in a compact manner which infer self-connection among the filler particles. Materials with these surface characteristics offer applications in media require density of high energy and energy storage equipment e.g., capacitors [31]. Some cracks and pores were also observed in the composite surface (Fig. 2b) which are considered to be produced during heat treatment (sintering) of the material as well as the low concentration of the filler particles [32].

\subsection{XRD Analysis}


XRD analysis was performed to identify the phase distribution and crystallinity of the prepared composite. In Fig. 3 , the XRD pattern $\left(2 \theta=13-70^{\circ}\right)$ represents two phase system of the material where initial peaks are for the PANI polymer while the rest of the peaks determine the CW phase of the composite. There occurs coexistence between CW particles and PANI polymer which confirms the composite production between the two partners [33]. This is due the interfacial interactions between PANI matrix and CW particles. Characteristic peaks of PANI polymer appear at $2 \theta=13-24^{\circ}$ which correspond to (110) and (200) lattice planes of the polymer [29]. Sharp peak at $2 \theta=26.65^{\circ}$ corresponds to (311) spinel crystallographic plane of $\mathrm{AB}_{2} \mathrm{O}_{4}$ type with a cubic geometry having a face-centered lattice which is usually found in ferrites [33]. The average crystallite size of CW/PANI was measured while using famous Scherer's equation [34] and was found to be $85.28 \mathrm{~nm}$ with a 0.0018 lattice strain. Broad and sharp peaks in XRD pattern of CW/PANI determined its semi-crystalline nature as reported in literature [29].

\subsection{Dielectric Properties}

Different dielectric properties of $\mathrm{CW}$ and CW/PANI were investigated to determine the charge and energy storage capacity of materials understudy at a specific frequency $(3 \mathrm{MHz}$ to $3 \mathrm{GHz})$ at ambient temperature.

\subsubsection{Dielectric constant $\left(\varepsilon^{\prime}\right)$}

The dielectric parameter $\varepsilon^{\prime}$ was measured using the following formula

$$
\epsilon^{\prime}=C d / \epsilon_{0} A
$$

1

In the expression, $C$ is the capacitance, $d$ id the pellet thickness, $\epsilon_{0}$ is permittivity of free space and $A$ is the pellet cross section area.

Figure 4 shows that dielectric constant ( $\left.\varepsilon^{\prime}\right)$ for both CW (inset plot) and CW/PANI composite has higher values at low frequency range. With increase in frequency, the dielectric constant decreases and pursue a constant value of $1.6 \mathrm{GHz}$ and further increase in frequency causes resonance. The reason for high $\varepsilon^{\prime}$ value at low frequency dispersion can be due to the space charge effects representing the charge carriers hopping between different metal ions present in the CW [35]. The presence of possible metallic electrodes in $\mathrm{CW}$ accumulates charges at CW/PANI interface which ultimately increases $\varepsilon^{\prime}$ at initial frequency region [36]. It has been found that at high frequency, polar sites in the composite system cannot adjust themselves so rapidly to respond. This results in dielectric relaxation and interfaces in the composite, consequently $\varepsilon^{\prime}$ decreases. In gigahertz frequency regions, dielectric relaxation produces due to the alternation of electric and elastic behavior and the movement of domain walls [36]. The dispersion of $\varepsilon^{\prime}$ as a function of frequency is attributed to the creation of Maxwell-Wagner kind of interfacial polarities [37]. From CW/PANI plot, it is clear that the composite has much higher $\varepsilon^{\prime}=137$ compared to pure CW $\varepsilon^{\prime}$ $=6.5$ (inset plot) at low frequency $(1.5 \mathrm{MHz})$ region. This is attributed to higher polarity of PANI matrix in the composite. Conversely, $\varepsilon^{\prime}$ of CW/PANI composite decrease considerably $\left(\varepsilon^{\prime}=1.70-0.10\right)$ with 
increasing frequency and by $2.38 \mathrm{GHz}$, PANI based CW composite exhibit similar $\varepsilon^{\prime}$ value just like pure $\mathrm{CW}$. This shows that at high frequency, the mobility of polar groups in PANI chains is too slow to contribute to $\varepsilon^{\prime}$ [38]. It seems that at high frequency, ceramic component dominates the $\varepsilon^{\prime}$ of CW/PANI composite. Concluding the summary, $\varepsilon^{\prime}$ of the CW/PANI depends on the dielectric characteristics of both ceramic and polymer constituents, while at high frequencies $\varepsilon^{\prime}$ depends primarily on the ceramic filler component of the composite.

\subsubsection{Dielectric loss (tanס)}

For designing capacitors, the magnitude of $\tan \delta$ acts as a significant factor. According to the literature [24] for a perfect capacitor, $\tan \delta$ should have the smallest possible value. The $\tan \delta$ was measured using the following expression 2 .

$$
\tan \delta=\frac{1}{2 \Pi f R_{p} C_{p}}
$$

2

where $f$ is the frequency of applied electric field and $C_{p}$ and $R_{p}$ are equivalent parallel capacitance and resistance respectively.

The variations of tand as a function of frequency at room temperature for $\mathrm{CW}$ and CW/PANI are given in Fig. 5 (a) and (b).

It is evident that $\tan \delta$ for $\mathrm{CW}$ is decreasing linearly with frequency sweep indicating insulation activity in ceramic powder. Slight increase at 1.8 and $2.4 \mathrm{GHz}$ is caused by polarization loss in CW. CW/PANI composite does not show any significant dielectric loss with increasing applied field frequency. The abrupt $\tan \delta$ upshift at $2.37 \mathrm{GHz}$ of the composite is an indication of the onset of CW contribution [38]. Dipole relaxation at high frequency regions also causes increase in loss tangent. Both $\mathrm{CW}$ powder and $\mathrm{CW} / \mathrm{PANI}$ composite have stable value of $\tan \delta$ and $\varepsilon^{\prime}$ in between $1 \mathrm{MHz}-2 \mathrm{GHz}$ range which is very significant in a number of applications. The low and stable dielectric loss of CW/PANI renders the composite to be one of the more active scaffolds for use in high frequency applications such as high range capacitors, UV detectors and microwave signal processing at high frequency [35].

\subsubsection{Capacitance}

Frequency dependent capacitance was also measured at room temperature as shown in Fig. 6 . For both $\mathrm{CW}$ and CW/PANI, a slight decrease in capacitance indicates the space region reduction near electrodes surface [39]. Capacitance value from $3.7 \mathrm{pF}$ to $1.6 \mathrm{pF}$ at $1.6 \mathrm{GHz}$ for CW/PANI is due to charge carriers that are comparatively blocked adjacent to electrodes surfaces. A consistency in the magnitude of capacitance supports the results of dielectric losses of the composite.

\subsubsection{AC conductivity}


$A C$ (alternating current) conductivity of CW powder as well as CW/PANI composite was studied at room temperature. Following equation 3 was used to determine $A C$ conductivity.

$$
\sigma_{a c}=\varepsilon^{\prime} \epsilon_{\square} \omega \tan \delta
$$

3

where $\sigma$ denotes conductivity, $\varepsilon^{\prime}$ is dielectric constant, $\epsilon_{\square}$ represents permittivity $\left(8.85 \times 10^{-12} \mathrm{Fm}^{-1}\right), \omega=$ $2 \pi f$ and $\tan \delta$. By putting all the given and calculated values in equation $3, \sigma_{a c}$ was measured for the materials under study (Fig. 7 (a) and (b)). At about $2 \mathrm{GHz}, \sigma_{a c}$ increases for both samples that infers the conductivity dominant dispersion regions at low frequency regions. However, further going onward from $2 \mathrm{GHz}$, a match in frequency (resonance) of the induced and applied fields happens.

It is evident that the conductivity of CW is smaller $\left(0.037-0.098 \mathrm{Scm}^{-1}\right)$ which has been enhanced by its insertion in the polymer matrix. The increase in $\sigma_{a c}$ for both $\mathrm{CW}\left(0.037-0.098 \mathrm{Scm}^{-1}\right)$ and CW/PANI (6.73 $-7.55 \mathrm{Scm}^{-1}$ ) with frequency is attributed to electronic polarization and hooping of charge carriers between the localized states. With this space charge fluctuation occurs through the interface which results in intrinsic conduction [40]. It means that $\sigma_{a c}$ dominates at high frequency regions. Moreover, PANI polarons play a considerable part in enhancing the conductive property in the composite. Comparative analysis of $\sigma_{a c}$ and other dielectric properties of the two materials is given in Table 2 .

Table 2. A comparative analysis of dielectric properties of the studied materials.

\begin{tabular}{lccc}
\hline Sample ID & $\partial_{a c}\left(\mathrm{Scm}^{-1}\right)$ & $\varepsilon^{\prime}$ (units) & $\tan \delta$ (units) \\
\hline CW & 0.098 & 8.30 & 1.31 \\
CW/PANI & 7.55 & 137 & 255 \\
\hline
\end{tabular}

The increase in conductivity with frequency is also supported by frequency versus resistance plots for $\mathrm{CW}$ and CW/PANI as shown in Fig. 8. Resistance (Rs) decreases with frequency sweep linearly which in turn reflects increase in conductivity of the materials. The enhanced conductivity of CW/APNI suggests that the composite could be prominent candidate for those technologies that require better conductivity for suppressing charge injection [41].

\subsubsection{Complex impedance (Cole-Cole plot)}

Figure $9(\mathrm{a}, \mathrm{b})$ represents Cole-Cole plot (complex impedance) for CW and CW/PANI respectively at 300K. Real and imaginary impedance ( $Z$ ' $Z \square$ respectively) were extracted from the following equation considering magnitudes of $|\mathrm{Z}|$ and phase angle $\theta$.

$$
Z^{\prime}=|Z| \cos \theta, Z^{\prime \prime}=|Z| \sin \theta
$$


The shape of the plot in both cases is a semi-circle that is usually noticed in materials having super-ionic characteristics [42]. At high frequency regions, the formation of semi-circle is due to interfacial effects that reflect single conduction mechanism in the samples. Here, the curves denote vast dipole relaxation while intercepts on $x$-axis display the resistance of material in bulk (Rb). Complex impedance shows decrease in $\mathrm{Rb}$ for the composite $(\mathrm{Rb}=4.490 \Omega$ ) as compared to the ceramic $(\mathrm{Rb}=8.492 \Omega)$. This verifies that the resistance phenomenon in the composite is decreasing with ceramic loading in the PANI matrix [43].

\section{Conclusions}

The present study is novel in the context of Peshawar Pakistan as the ceramic industries here are producing huge amount of solid wastes causing various types of environmental pollution. No work has been done here at Pakistan in respect of the incorporation of ceramic wastes in PANI matrix which confirms its originality. Industrial ceramic wastes were effectively encapsulated in PANI polymer media using in-situ (addition) free radical polymerization route to obtain CW/PANI composite. Instead of strong organic solvents, water was used as solvent for making solutions to make synthesis procedures more eco-friendly. Elemental composition of CW was done by EDX analysis. Morphology of CW powder and CW/PANI composite was obtained by using SEM analysis. Extensive dielectric analysis was carried out and it was found that the composite could serve as a satisfying source in electro-responsive technologies for better conductivity demand as well as photo detector materials.

\section{Declarations}

\section{Acknowledgement}

We appreciate research and Lab support provided by NCE in Physical Chemistry and Institute of Chemical Sciences, University of Peshawar.

\section{Conflict of interest}

The authors have no conflict of interest regarding the submission of this article.

\section{References}

1. Alshemmari, H., Alotaibi, Y., R.J.K.J.S, E. \& OwensTrace metal concentrations in the surface sediments of Sulaibikhat Bay, Kuwait.2010. 37(2A):p. 87-110

2. Chen, H. J., Yen, T. \& Chen, K. H. Use of building rubbles as recycled aggregates. Cement and concrete research, 33 (1), 125-132 (2003).

3. Topcu, I. \& Canbaz, M. Utilization of crushed tile as aggregate in concrete. 2007 
4. Pacheco-Torgal, F. \& Jalali, S. J. C. and b. materials, Reusing ceramic wastes in concrete. 2010. 24(5): p. 832-838

5. Raval, A. D. et al. Re-use of ceramic industry wastes for the elaboration of eco-efficient concrete. 2013. 2(3): p. 103-105

6. Toledo Filho, R. et al. Potential for use of crushed waste calcined-clay brick as a supplementary cementitious material in Brazil. 2007. 37(9): p.1357-1365

7. Gonçalves, J. et al. Performance evaluation of cement mortars modified with metakaolin or ground brick. 2009. 23(5): p.1971-1979

8. Sánchez de Rojas, M. I. et al.Properties and performances of concrete tiles containing waste fired clay materials.2007. 90(11):p. 3559-3565

9. Silva, J. et al. Fine ceramics replacing cement in mortars Partial replacement of cement with fine ceramics in rendering mortars. 2008. 41(8): p.1333-1344

10. Frías, M. et al. Properties of calcined clay waste and its influence on blended cement behavior. 2008. 91(4): p.1226-1230

11. Sánchez de Rojas, M. I. et al. Morphology and properties in blended cements with ceramic wastes as a pozzolanic material. 2006. 89(12): p.3701-3705

12. Sun, Z. et al. Synthesis and thermal behavior of geopolymer-type material from waste ceramic. 2013. 49: p. $281-287$

13. Mukharjee, B. B., Barai, S. V. J. W. M. \& ResearchDevelopment of construction materials using nanosilica and aggregates recycled from construction and demolition waste.2015. 33(6):p. 515-523

14. Rakhshani, A. et al. Preparation and characterization of nitrogen doped ZnO films and homojunction diodes. 2014. 41(1)

15. Zimmermann, M. V. \& Zattera, A. J. J. W.Recycling and reuse of waste from electricity distribution networks as reinforcement agents in polymeric composites.2013. 33(7):p. 1667-1674

16. Cao, J. et al. Recycling of waste fly ash for production of porous mullite ceramic membrane supports with increased porosity. 2014. 34(13): p.3181-3194

17. Gök, A., Omastova, M. \& Prokeš, J. J. E. P. J. Synthesis and characterization of red mud/polyaniline composites: electrical properties and thermal stability. 2007. 43(6): p.2471-2480

18. Kanwal, F. et al. Synthesis of coral-like silver chloride-polypyrrole nanocomposites derived from silver nanoparticles and the study of their structural, thermal, optical and electrical properties. 2021. 48(4)

19. Aman, A. et al. Polymeric composite based on waste material for high voltage outdoor application. 2013. 45(1): p.346-352

20. Khalil, A. et al. Effect of some waste additives on the physical and mechanical properties of gypsum plaster composites. 2014. 68: p. 580-586

21. Farooqi, B. A. et al. Graphene-polyaniline composite as superior electrochemical sensor for detection of cyano explosives. 2020. 138: p. 109981 
22. Sayed, M. A. et al. Photocatalytic hydrogen generation from raw water using zeolite/polyaniline@ Ni2O3 nanocomposite as a novel photo-electrode. 2019. 187: p.115943

23. Senguttuvan, S. et al. Significance of conducting polyaniline based composites for the removal of dyes and heavy metals from aqueous solution and wastewaters-A review. 2020: p. 129201

24. Khan, M. S. et al. Industrial ceramic waste in Pakistan, valuable material for possible applications. 2016. 139: p. 1520-1528

25. Kamarudin, S. et al. Investigation on size and conductivity of polyaniline nanofiber synthesised by surfactant-free polymerization. 2021. 14: p.255-261

26. Nepomuceno, N. et al. Evaluation of conductivity of nanostructured polyaniline/cellulose nanocrystals (PANI/CNC) obtained via in situ polymerization. 2021: p. 122372

27. Liu, P., Qiu, J. \& Wu, X. J. M. L. Well-defined polyaniline nanotubes and nanofibers surface-modified with poly (methyl methacrylate) via in-situ radical polymerization. 2012. 77: p. 4-6

28. Liu, P., Qiu, J. \& Wu, E. X.J.J.o.t.T.I.o.CSynthesis of polyaniline nanorods/polystyrene composite via facile in situ radical bulk polymerization.2013. 44(4):p. 686-690

29. Khan, M. S. et al. Synthesis and characterization of three-phase polymer-graphene oxide-ceramic composites. J. Chem. Soc. Pak, 38 (02), 234 (2016).

30. Von Tumacder, D. et al. Electropolymerized polypyrrole/safranin-O films: Capacitance enhancement. 2021: p. 124099

31. Malana, M. A. et al. Synthesis, structural, magnetic and dielectric characterizations of molybdenum doped calcium strontium M-type hexaferrites. 2016. 42(2): p.2686-2692

32. Voleppe, Q. et al. Enhanced fracture resistance of thermoset/thermoplastic interfaces through crack trapping in a morphology gradient. 2021. 218: p.123497

33. Khursheed, T. et al. Synthesis and characterization of polyaniline-hexaferrite composites. 2015. 393: p. 8-14

34. Sohail, M. et al. Synthesis and Characterization of Mg-Matrix Based TiO 2/Al 203 Composite Materials. 2021: p. 1-10

35. Chouaibi, H. \& Khirouni, K. and E.J.P.i.N.S.M.I. Dhahri, Enhanced dielectric performance of Dysubstituted YMn205 for high-frequency applications. 2021

36. Badapanda, T. et al. Structural and dielectric properties of polyvinyl alcohol/barium zirconium titanate polymer-ceramic composite. 2013. 13(7): p. 1490-1495

37. Ramana, C. et al. Correlation between structural, magnetic, and dielectric properties of manganese substituted cobalt ferrite. 2013. 114(18): p.183907

38. Popielarz, R. et al. Dielectric properties of polymer/ferroelectric ceramic composites from $100 \mathrm{~Hz}$ to $10 \mathrm{GHz}$. 2001. 34(17): p.5910-5915

39. Al-Haddad, R. M. et al. DC and AC conductivity measurements of Se60Te30Ge10 films. 2009. 12(4): p. 72-77

40. Solymar, L., Walsh, D. \& Syms, R. R. Electrical properties of materials (Oxford university press, 2014). 
41. Cheng, S. et al. Polymer Dielectrics Sandwiched by Medium-Dielectric-Constant Nanoscale Deposition Layers for High-Temperature Capacitive Energy Storage. 2021

42. Belattar, J. et al.Electric modulus-based analysis of the dielectric relaxation in carbon black loaded polymer composites.2010. 107(12):p. 124111

43. Khan, M. S. et al. Synthesis and characterization of three-phase polymer-graphene oxide-ceramic composites. 2016. 38(02): p. 234

\section{Figures}

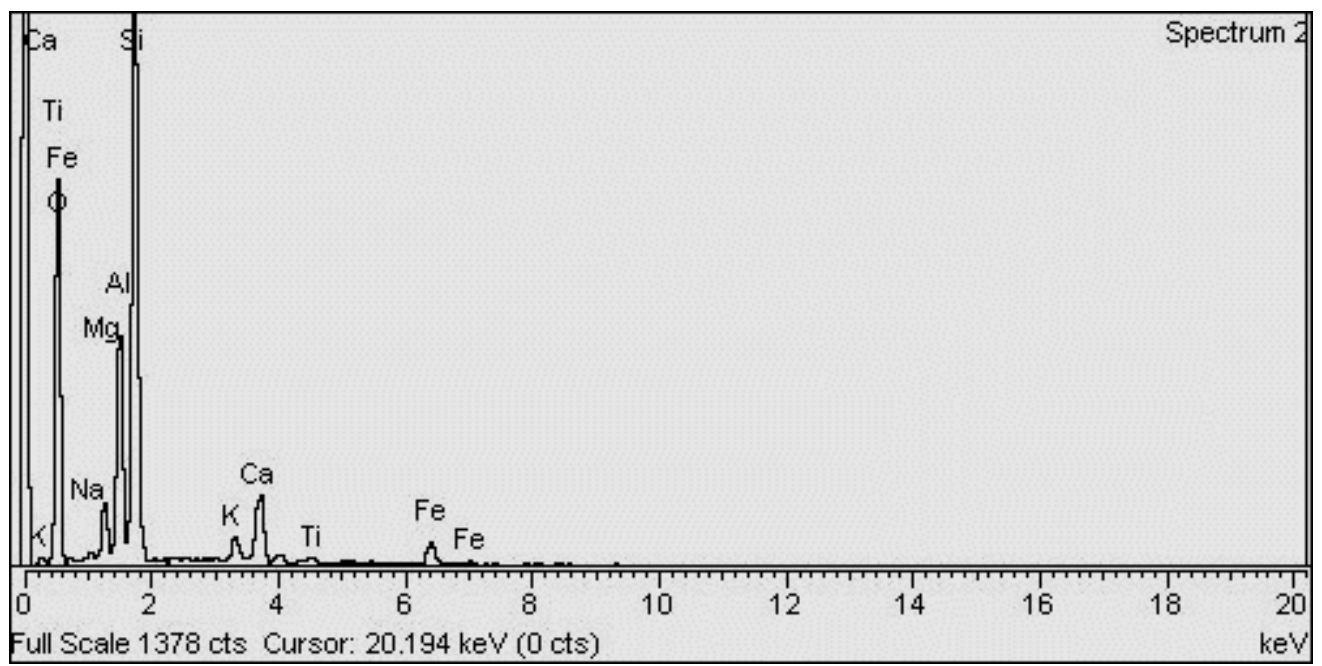

\section{Figure 1}

EDX spectrum of CW. 


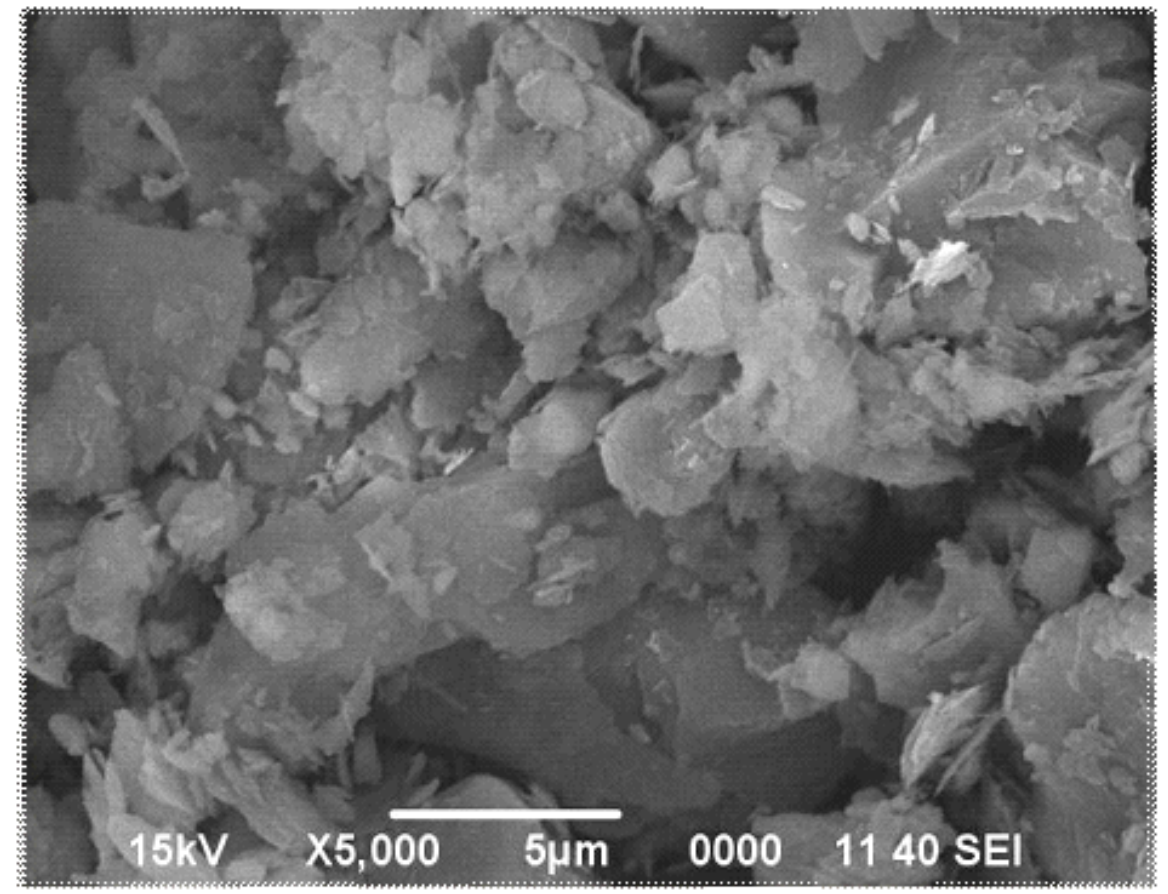

(a)

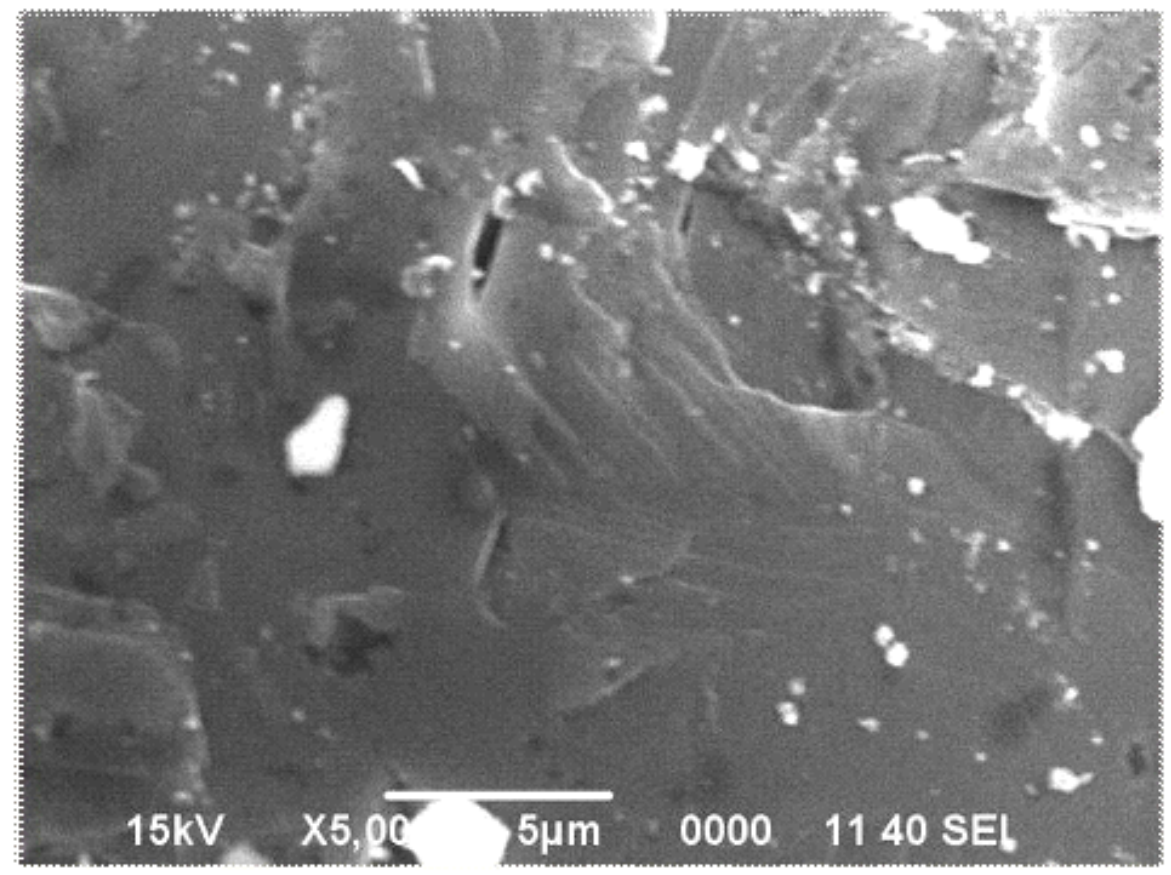

(b)

Figure 2

SEM micrographs of (a) CW (b) CW/PANI. 


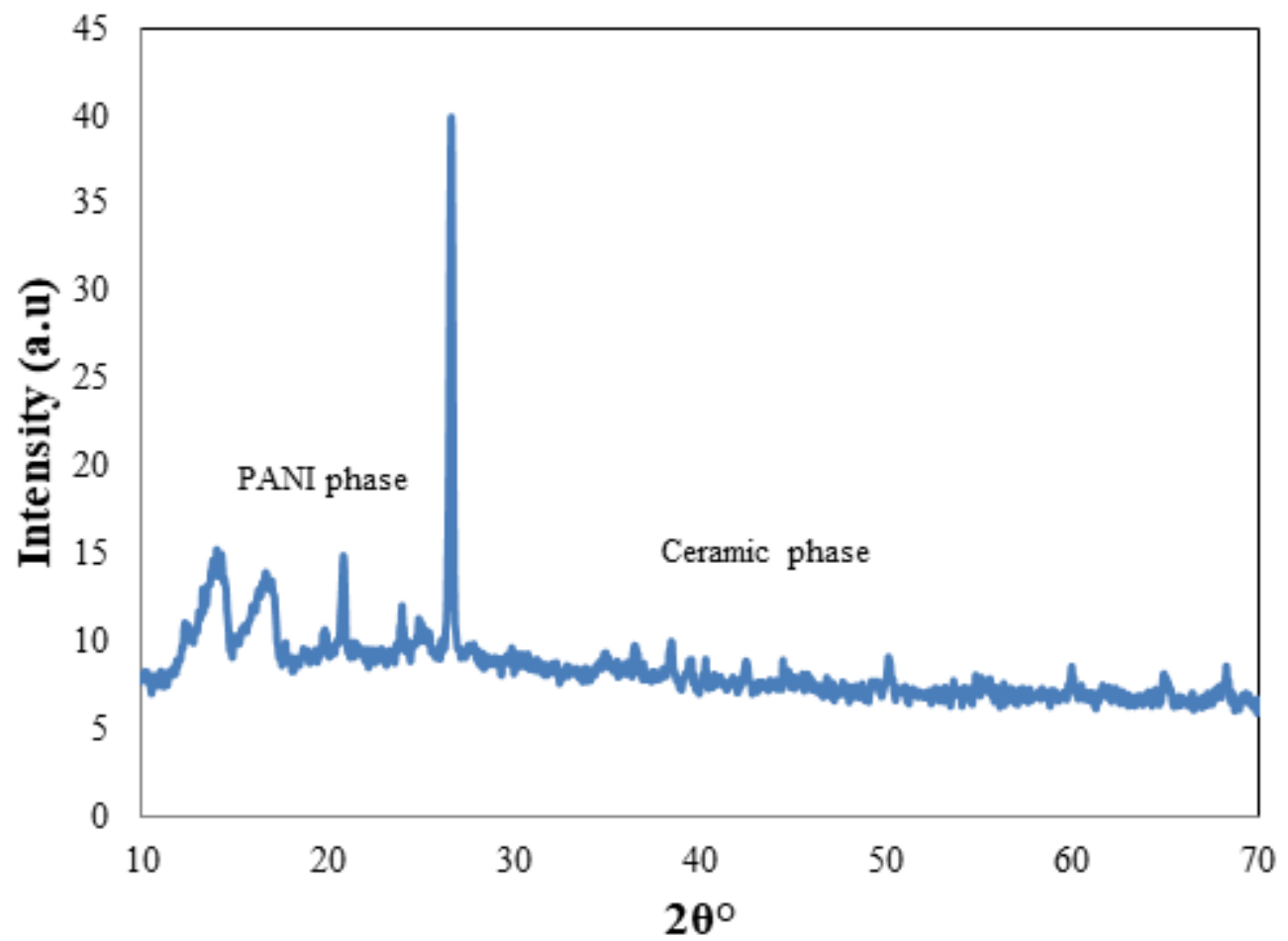

Figure 3

XRD patterns of CW/PANI composite material.

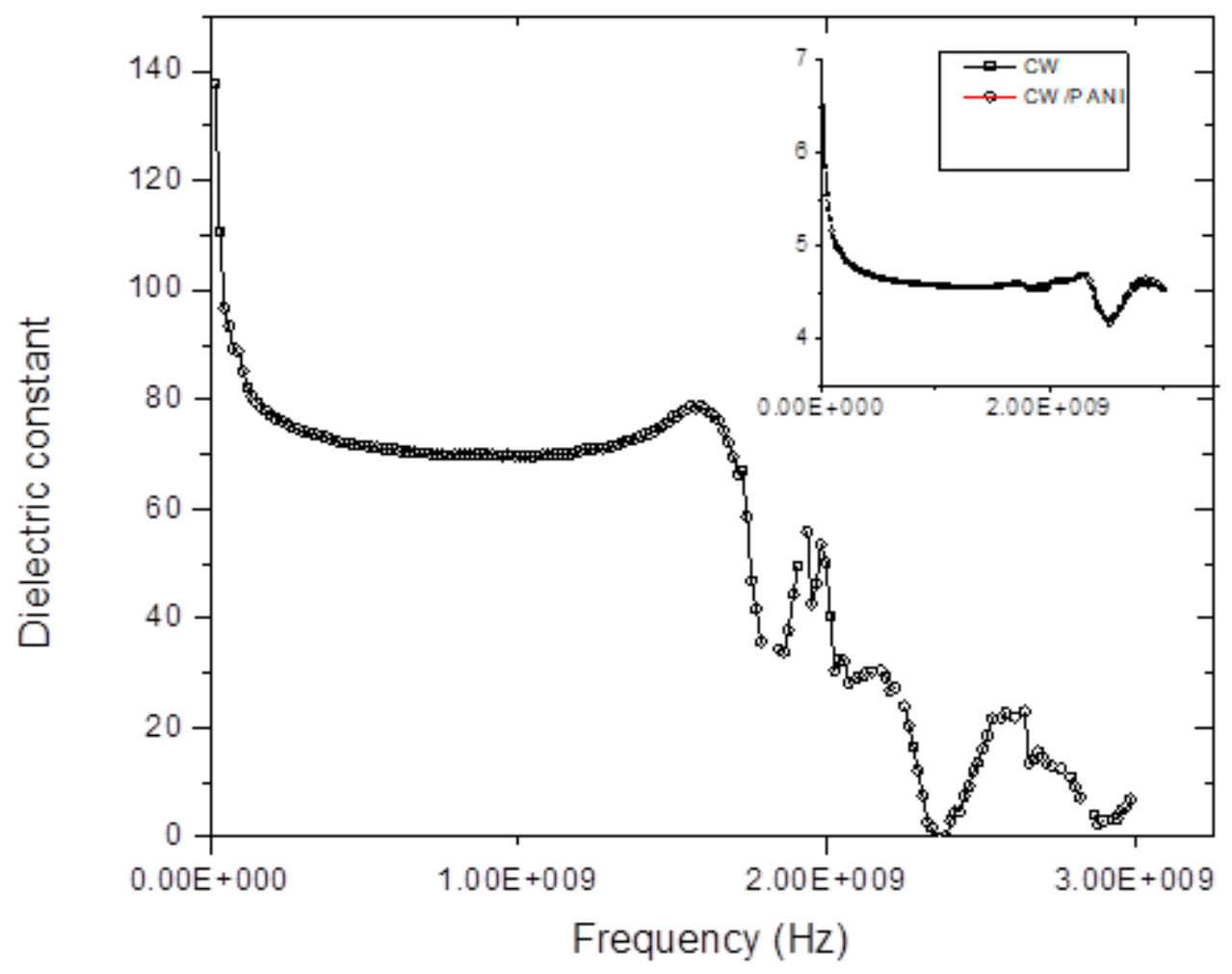


Figure 4

Dielectric constant as a function of frequency at $300 \mathrm{~K}$ for CW/PANI. (The inset plot shows dielectric constant for $\mathrm{CW}$ )
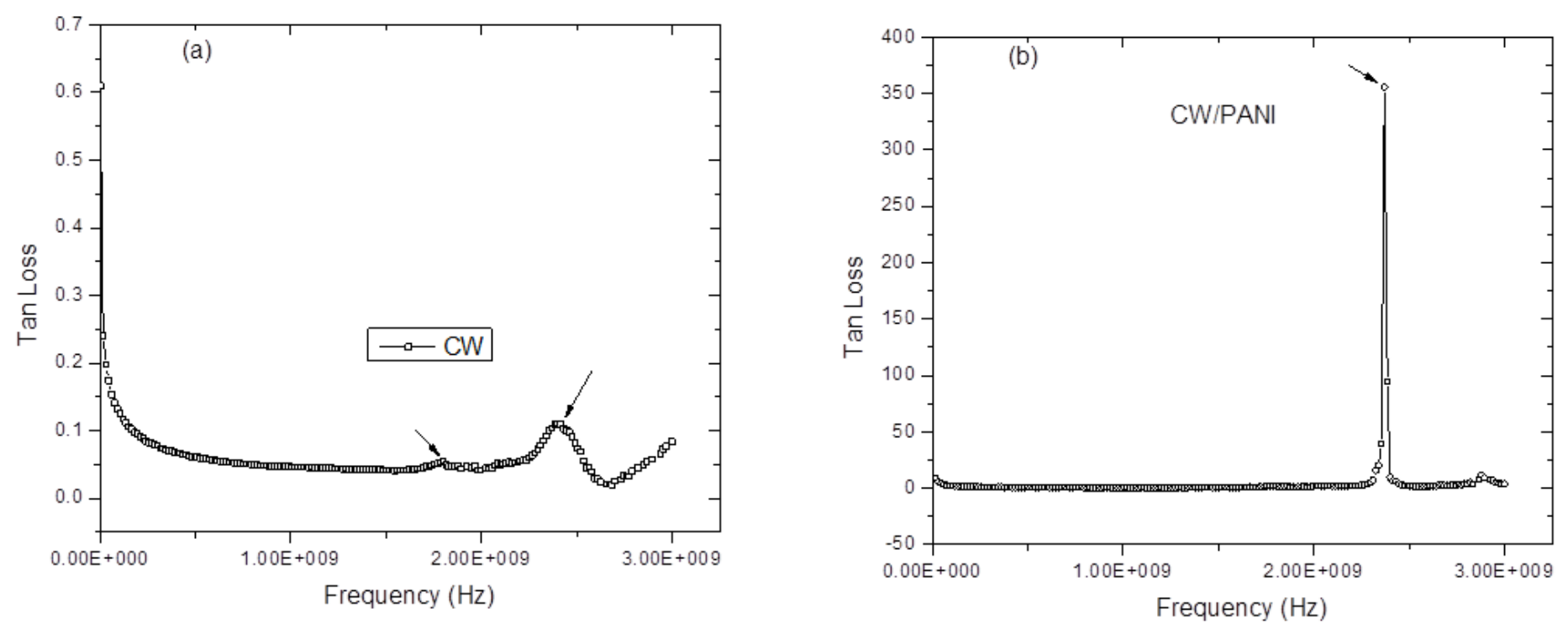

\section{Figure 5}

Frequency dependent dielectric loss at 300K (a) CW (b) CW/PANI.

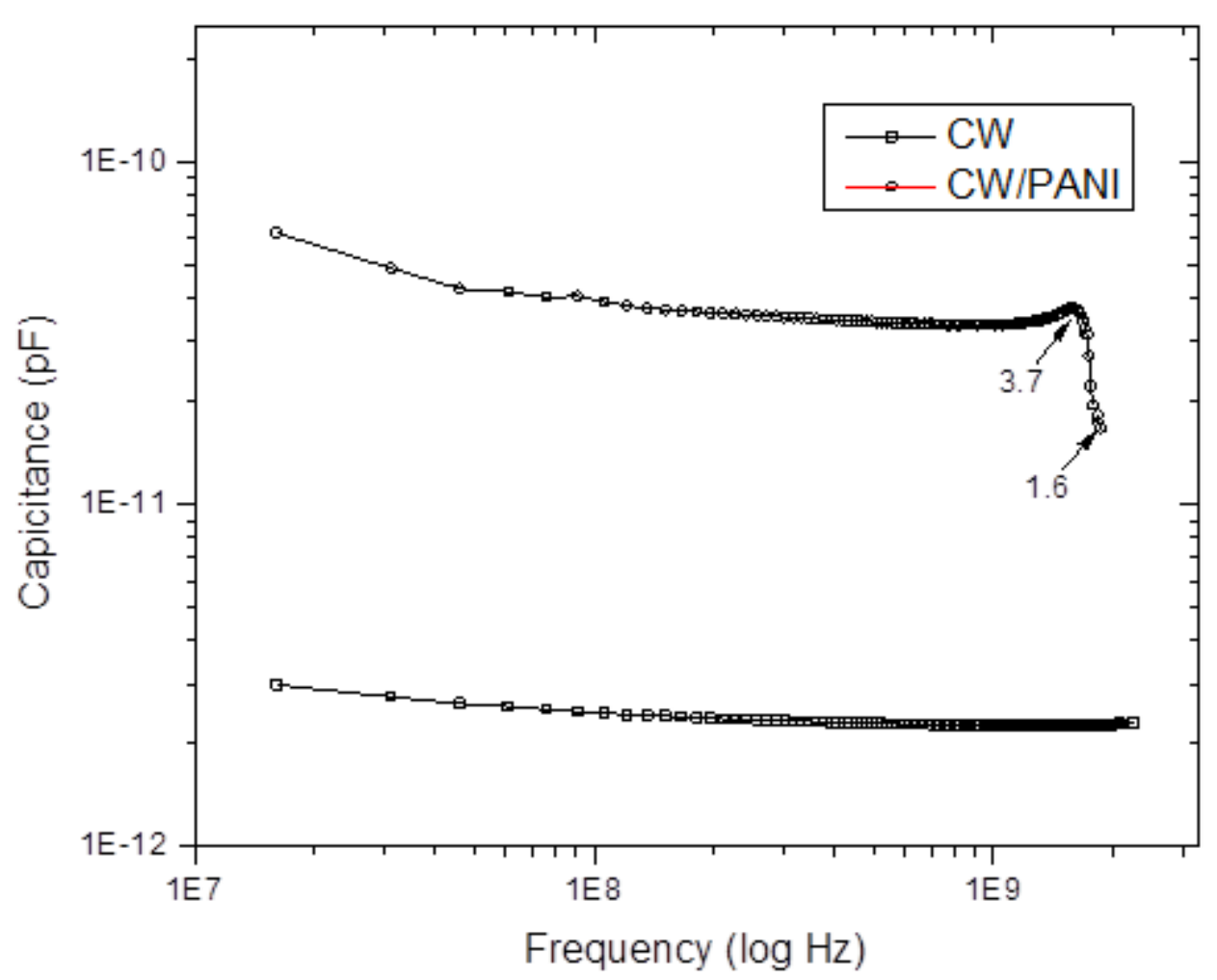


Figure 6

Variation in capacitance of CW and CW/PANI with frequency.
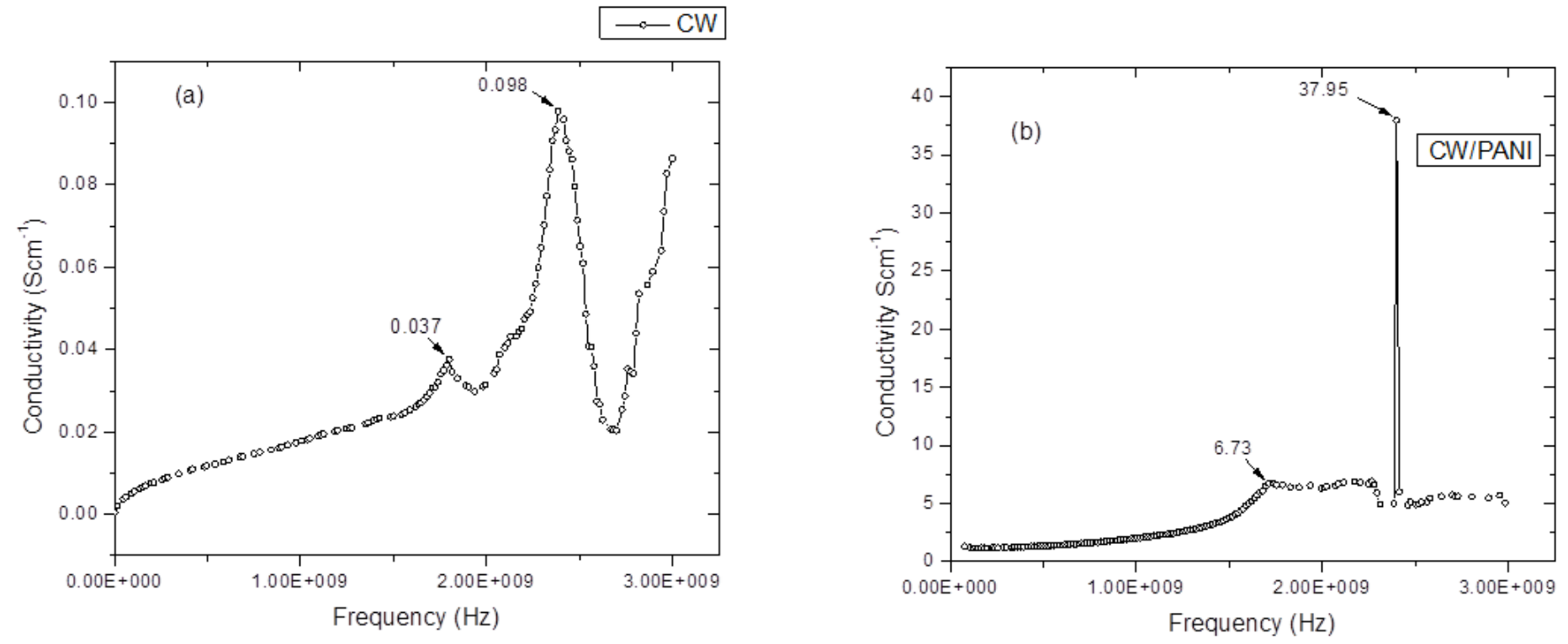

Figure 7

AC conductivity as function of frequency at $300 \mathrm{~K}$ (a) CW (b) CW/PANI.

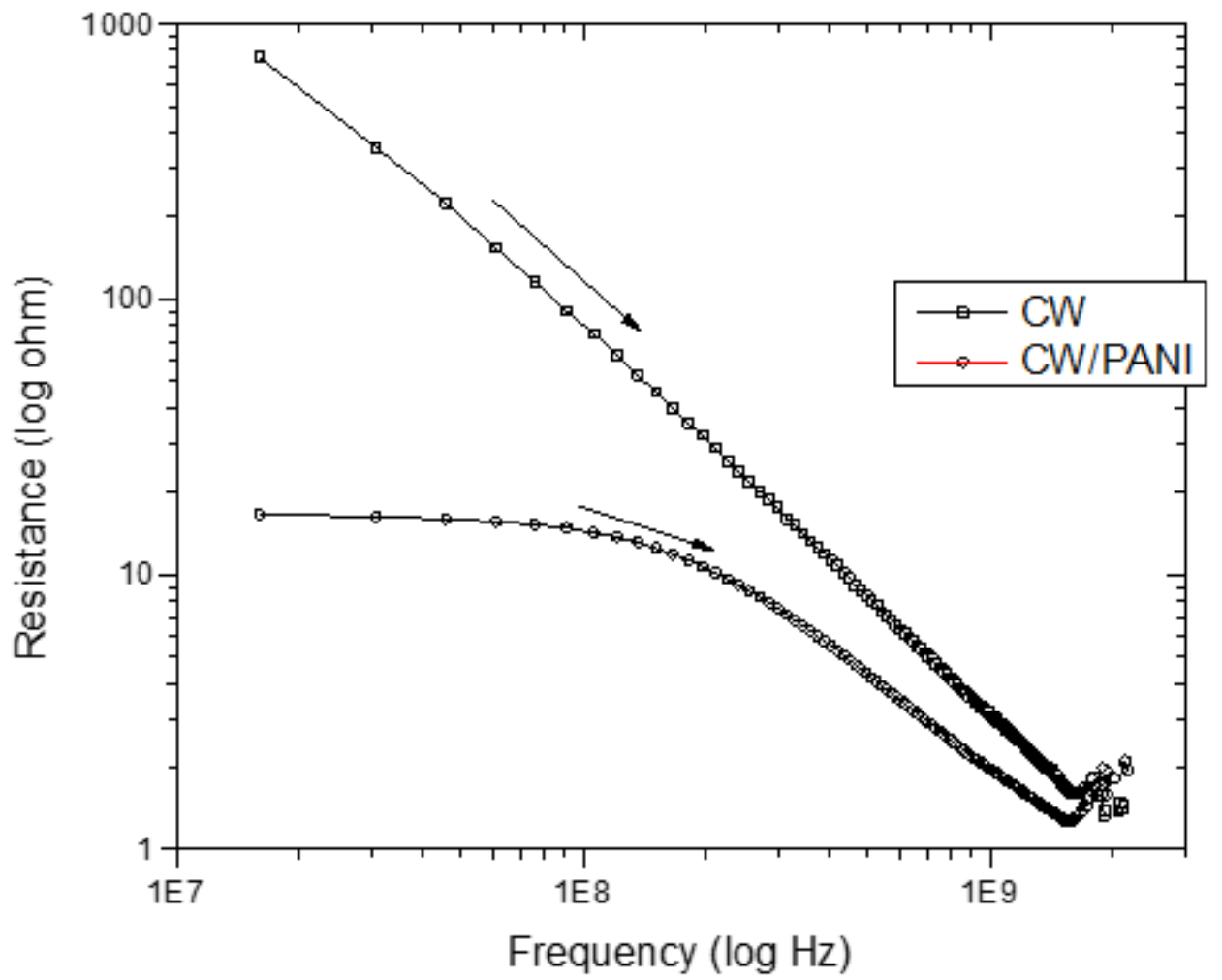


Figure 8

Frequency versus resistance plot for $\mathrm{CW}$ and CW/PANI samples.
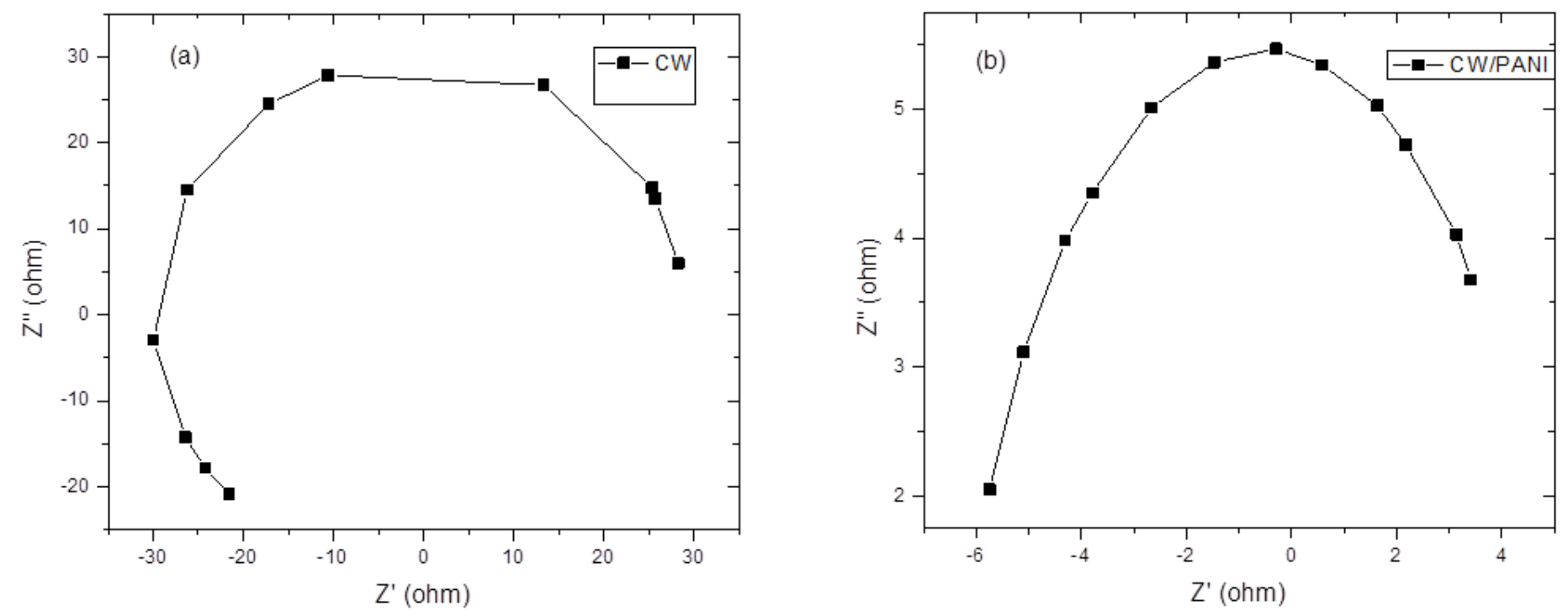

Figure 9

Cole-Cole plots of complex impedance (Z\vs. Z\) of the samples as a function of frequency. 